\title{
Temperature Measurement Using Passive Harmonics during High Intensity Focused Ultrasound Exposures in Porcine Tissue
}

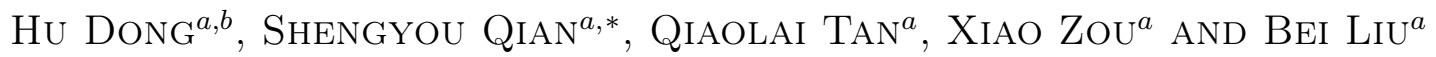

${ }^{a}$ College of Physics and Information Science, Hunan Normal University, Changsha 410081, PR China

${ }^{b}$ Department of Information and Engineering, Changsha Normal University, Changsha 410100, PR China

(Received February 26, 2018; in final form July 14, 2018)

It is important to deliver suitable thermal dose to target region during high intensity focused ultrasound therapy,and ultrasonic non-invasive temperature measurement is an easily accepted approach to monitor the temperature fields during the treatment process. Traditional method usually required another source for monitoring besides high intensity focused ultrasound source. This paper was based on passive scattering harmonics for high intensity focused ultrasound temperature measurement, and it could avoid the influence of treatment sources on temperature measurement. The purpose of this paper is to investigate the relationship between the nonlinear harmonic amplitude and temperature in porcine tissue (muscle and liver). The optical fiber hydrophone was used to receive passive scattering harmonics from high intensity focused ultrasound irradiation in porcine tissue, and the temperature of tissue in focal region was measured with a thermocouple needle at the same time. The relationship between harmonic amplitude and temperature was analyzed. Experimental results indicate that the values of amplitude for fundamental wave $P_{1}$, the second harmonic $P_{2}$, the third harmonic $P_{3}$, the ratios of $P_{2} / P_{1}, P_{3} / P_{1}$, and $P_{3} / P_{2}$ decrease while temperature increases in the focal region of porcine tissue, and the slope of $P_{2} / P_{1}$, $P_{3} / P_{1}$, and $P_{3} / P_{2}$ have obvious decline at the beginning of temperature measurement, but their slope become flat as temperature increases, and the slope of $P_{2} / P_{1}, P_{3} / P_{1}$, and $P_{3} / P_{2}$ drop faster in porcine muscle than in liver. This study may potentially be used as an non-invasive method of temperature monitoring for ultrasonic thermal therapy in real-time.
\end{abstract}

DOI: 10.12693/APhysPolA.134.524

PACS/topics: high intensity focused ultrasound, temperature measurement, passive harmonic

\section{Introduction}

High intensity focused ultrasound (HIFU) therapy is used to achieve therapeutic effect mainly by its thermal effect. Ultrasonic energy can be used to make the temperature of tumor cells elevated to $45^{\circ} \mathrm{C}$ or higher to inhibit tumor cells growth [1]. In order to safely and accurately kill the pathological tissue but do not damage normal tissue, it is necessary to monitor the process of treatment. In clinical treatment, the therapeutic dose of HIFU may be excessive and it may cause severe burn to skin and normal tissue, or it may lack effective monitoring in real-time, and this might affect tumor cells treatment. In order to guarantee the safety and reliability of HIFU therapy, the technology of temperature monitoring has become a hot topic in medical ultrasound engineering.

During the heat treatment process of cancer, the monitoring method can be achieved by temperature measurement, which includes non-invasive and invasive temperature measurement. The invasive temperature measurement inserts temperature sensors into the test site for direct measurement and it could cause damage to tissue. There are many methods of non-invasive temperature

\footnotetext{
*corresponding author; e-mail: syqian@foxmail.com
}

measurement, such as MRI and ultrasound, etc. Temperature measurement with MRI is based on the shift of neutron resonance frequency $[2,3]$. The advantage of this method is that the temperature distribution in the treatment area can be measured within a short time $(5 \mathrm{~s})$, and the dynamic temperature of the treatment area can also be obtained by continuous measurement at regular intervals. On the other hand, this method is too expensive and more complex. Ultrasound is penetrative and easily compatible with treatment equipment [4-6]. Temperature measurement with ultrasound included two different ways of ultrasonic signal and image, and their fundamental principles are all the relationship between the acoustical properties of biological tissue and temperature. From ultrasonic signal, temperature measurement is mainly based on ultrasonic sound speed, nonlinear parameter $B / A$, time shift of echo, frequency shift of echo, and scattering energy, etc. From ultrasonic image, temperature measurement is mainly based on image texture and image gray-scale [7]. All these ultrasonic methods mentioned above required a diagnostic ultrasound source for monitoring, and the method proposed in this paper is directly based on HIFU source and nonlinear characteristics of HIFU.

The ultrasound wave with finite amplitude is gradually distorted in the propagation process, which can produce high order harmonics and they can be detected in the frequency spectrum of signal. According to the simulation 
of sound pressure and transmission distance with transducer, ultrasonic distortion and harmonics generation depend on $B / A$ parameter, attenuation coefficient, and acoustic speed in media $[8,9]$. Because these three parameters are relatively easy to be influenced by temperature, so harmonics are correlated with temperature. In a numerical simulation study, van Dongen and Verweij [10] found that ultrasonic amplitude in glycerol was related to temperature. They proved that temperature can be measured by amplitude ratio between fundamental wave and second harmonic. In this paper, the scattering harmonics came from HIFU irradiation in porcine muscle and liver, and they were passively received by a fiber hydrophone. A thermocouple needle was used to monitor focal region temperature of porcine muscle and liver.

\section{Materials and methods}

\subsection{Experimental setup}

The experimental porcine tissue(muscle and liver) were fresh,and large blood vessels and meridians were avoided.The tissue was cut to a size of around $6 \mathrm{~cm} \times 7 \mathrm{~cm} \times 4 \mathrm{~cm}$. The povidone and $95 \%$ alcohol were mixed with 1:4 proportion to scavenge oxygen in water, then mixing them and water with 1:20 proportion and pour them into the water tank, waiting for about one hour before experiment. The porcine tissue was mounted on a sample holder and immersed in water under HIFU source (PRO2008, Shenzhen, CN). Acoustic rubber was placed at the bottom of water tank to absorb ultrasound, and the position of transducer can be shifted by computer controlled 3D mobile platform. In the experiment, the HIFU source was self-focusing transducer with concave spherical surface, and it had a circular hole at the top that allowed the fiber probe of hydrophone (FOPH2000, Leutenbach, DE)and type-B mode ultrasound probe to pass through. The center frequency of self-focusing transducer was $1.39 \mathrm{MHz}$ and the geometric focal length was $13 \mathrm{~cm}$, the aperture of transducer was $11 \mathrm{~cm}$ and the diameter of circular hole was $4.7 \mathrm{~cm}$, and the source pressure of transducer was $0.23 \mathrm{MPa}$. The input electric power of transducer can be adjusted during experiment.

All exposures were accomplished using quasicontinuous HIFU wave and its pulse length was $10 \mathrm{~ms}$. The average temporal power of transducer can be measured by radiation force balance method as follows [11]:

$$
P=2 F c /\left(\cos \alpha_{1}+\cos \alpha_{2}\right),
$$

where $P$ was the average temporal power, $F$ was the radiation force, $c$ was the acoustic speed in propagating medium, $\alpha_{1}$ was half of the internal hole aperture angle of the self-focusing transducer, and $\alpha_{2}$ was half of the outer aperture angle of the self-focusing transducer.

The spatially peak acoustic intensity $I_{S P}$ was calculated by the equation [12]:

$$
I_{S P}=1.56 \frac{P}{D^{2}},
$$

where $D$ was the focal beam width of transducer. The average temporal power of transducer was $290 \mathrm{~W}$, the ellipsoidal $-6 \mathrm{~dB}$ focal beam width of this transducer was $1.6 \mathrm{~mm}$.

The scattering acoustic signal was received by hydrophone, then the signal was amplified by broadband amplifier and sent into digital oscilloscope (Tektronix MDO3032, USA) for observation, and signal was also stored and processed in computer. The thermocouple (DT-3891G, Shenzhen, CN) needle was placed in the focal region of porcine tissue during HIFU irradiation and the temperature was measured in real-time. The intensity and time of HIFU irradiation can be controlled by computer. The detection device of harmonics was shown in Fig. 1. The experiments were repeated 6 times at each temperature.

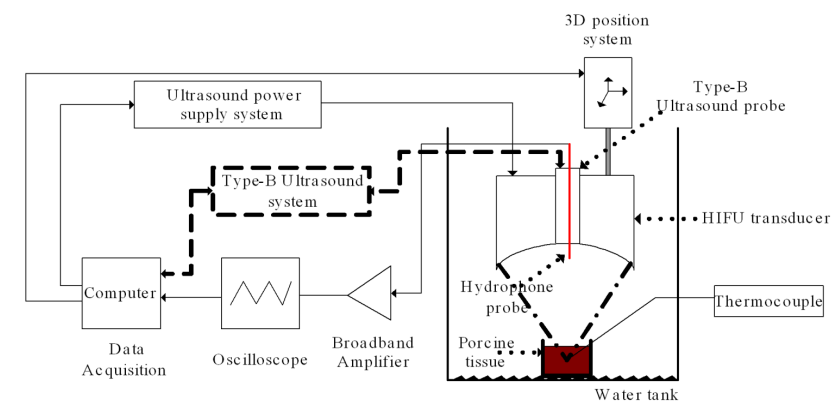

Fig. 1. Passive detection device of scattering harmonics.

\subsection{Data processing}

The scattering harmonics were analyzed and processed with Matlab programming. Firstly, the noise in ultrasonic signal was filtered out by sym8 wavelet with 4 layer decomposition, then we used signal processing box FDATool to design bandpass filter and made harmonics pass through. Because of the rapid attenuation of high order harmonic amplitude, the designed filter and data analysis were no more than third-order harmonics. Furthermore, fast Fourier transform (FFT) transform was performed for the scattering signals with the Hanning window. $P_{1}, P_{2}$, and $P_{3}$ are corresponding to fundamental wave, second harmonic, and third harmonic respectively, which came from HIFU irradiation in porcine tissue. Harmonic amplitude was acquired by calculating frequency spectrum of ultrasonic pulse with the Fourier transform. Both harmonic amplitude and the ratios of harmonic amplitude $\left(P_{2} / P_{1}, P_{3} / P_{1}\right.$, and $\left.P_{3} / P_{2}\right)$ with temperature variation in focal region of porcine tissue were analyzed.

\section{Results}

From Figs. 2 and 3, it can be found that the harmonic amplitude of $P_{1}, P_{2}$, and $P_{3}$ decrease while temperature increases in porcine muscle and liver, and the amplitude of high order harmonic is smaller than low order harmonic at certain temperature. 


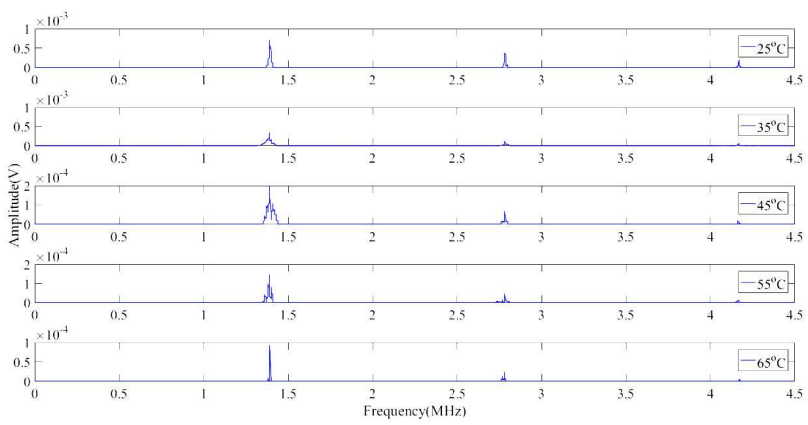

Fig. 2. Amplitude spectrum for the scattering HIFU signal in focal region of porcine muscle at $25^{\circ} \mathrm{C}-65^{\circ} \mathrm{C}$.

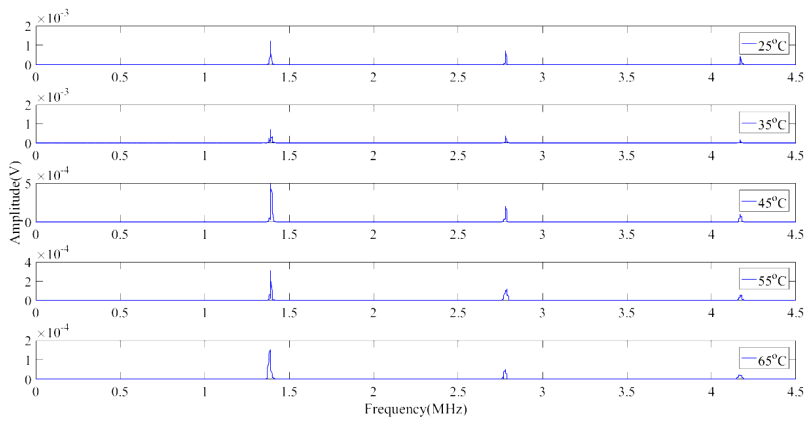

Fig. 3. Amplitude spectrum for the scattering HIFU signal in focal region of porcine liver at $25^{\circ} \mathrm{C}-65^{\circ} \mathrm{C}$.
Figure 4 shows the variation rate and standard deviation of harmonic amplitude $\left(P_{1}, P_{2}\right.$, and $\left.P_{3}\right)$ at different temperature in porcine muscle and liver. The variation rate is actually a relative value comparing to $25^{\circ} \mathrm{C}$, which can be decided with the variation divided by the amplitude at $25^{\circ} \mathrm{C}$. It can be found that the descending gradient of harmonic amplitude is bigger in porcine muscle than in liver, because the attenuation in muscle is greater than in liver $[13,14]$. With the increase of temperature, the difference of variation rate of harmonic amplitude decrease in porcine muscle and liver. Keck and Beyer [15] confirmed that the amplitude of $n$-order harmonic was proportional to $\left[\beta /\left(\alpha \rho c^{3}\right)\right]^{n-1}(\beta \alpha$ and $\rho$ denote nonlinear coefficient, attenuation coefficient, and density, respectively) during the propagation of finite amplitude wave in attenuation medium. The ultrasonic parameters which correlated with temperature are more prominent to the amplitude of high order harmonics, and the acoustic damping of high order harmonics is faster, so its amplitude is relatively low. Figure 5 shows the ratio $\left(P_{2} / P_{1}, P_{3} / P_{1}\right.$, and $\left.P_{3} / P_{2}\right)$ and standard deviation of various harmonic amplitude at different temperature in porcine muscle and liver, and the slope of $P_{2} / P_{1}, P_{3} / P_{1}$, and $P_{3} / P_{2}$ have obvious decline at the beginning of temperature measurement, but their slope becomes flat as temperature increases, and the slope of $P_{2} / P_{1}, P_{3} / P_{1}$, and $P_{3} / P_{2}$ drop faster in porcine muscle than in liver.
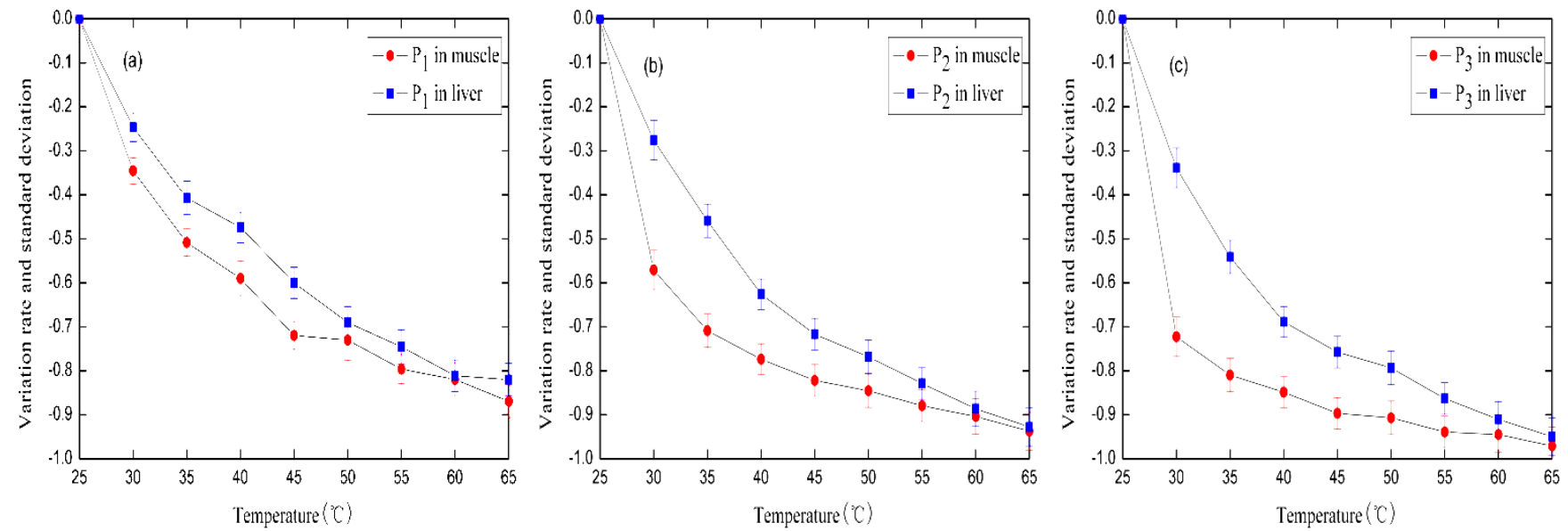

Fig. 4. Variation rate and standard deviation of harmonic amplitude at different temperature comparing to $25^{\circ} \mathrm{C}$ in porcine muscle and liver. (a) $P_{1}$, (b) $P_{2}$, (c) $P_{3}$.

From Figs. 2 to 5, it can be seen that harmonic amplitude decreases gradually while temperature increases in the focal region of porcine muscle and liver under the frequency of $1.39 \mathrm{MHz}$, this is mainly correlated with nonlinearity and absorption of ultrasound. The Gol'dberg number is expressed as $\Gamma=N / A$ [16] ( $N$ is related with nonlinearity and it is defined as $N=2 \pi f p_{0} f b e \beta, f$ is the center frequency of transducer and $p_{0}$ is the source pressure of transducer. $A$ is related with absorption and it is defined as $A=\rho c^{3} \alpha$ ), which defines the balance between nonlinearity and absorption process, the effect of nonlinearity is more obvious than absorption as $\Gamma>1$, but the effect of absorption is more obvious than nonlinearity as $\Gamma<1$. As the temperature increases in porcine muscle and liver, the increase of attenuation coefficient which decreases the generation of harmonics and the increase of ultrasonic nonlinearity enhances the number of wave distortion [17-19]. The values of physical constants used for porcine tissue are as follows: $\rho_{m}=1060 \mathrm{~kg} \mathrm{~m}^{-3}$, $c_{m}=1550 \mathrm{~m} \mathrm{~s}^{-1}, \alpha_{m}=6.1 \mathrm{~Np} \mathrm{~m}^{-1}, \beta_{m}=4.55$ 
for muscle and $\rho_{l}=1050 \mathrm{~kg} \mathrm{~m}^{-3}, c_{l}=1596 \mathrm{~m} \mathrm{~s}^{-1}$ $\alpha_{l}=4.5 \mathrm{~Np} \mathrm{~m}^{-1}, \beta_{l}=6.0$ for liver at $37^{\circ} \mathrm{C}[9,20$ 22]. For $1.39 \mathrm{MHz}$ ultrasound, the Gol'dberg number is about 0.26 and 0.43 for porcine muscle and liver at $37^{\circ} \mathrm{C}$, respectively. It means that the absorption effect is more obvious in porcine muscle than in liver at $35^{\circ} \mathrm{C}$, and we can find that the harmonic amplitude is smaller in porcine muscle than in liver at $35^{\circ} \mathrm{C}$ in Figs. 2 and 3. As a result, combining the effect of nonlinearity and absorption with temperature, the harmonic amplitude decreases while temperature increases.
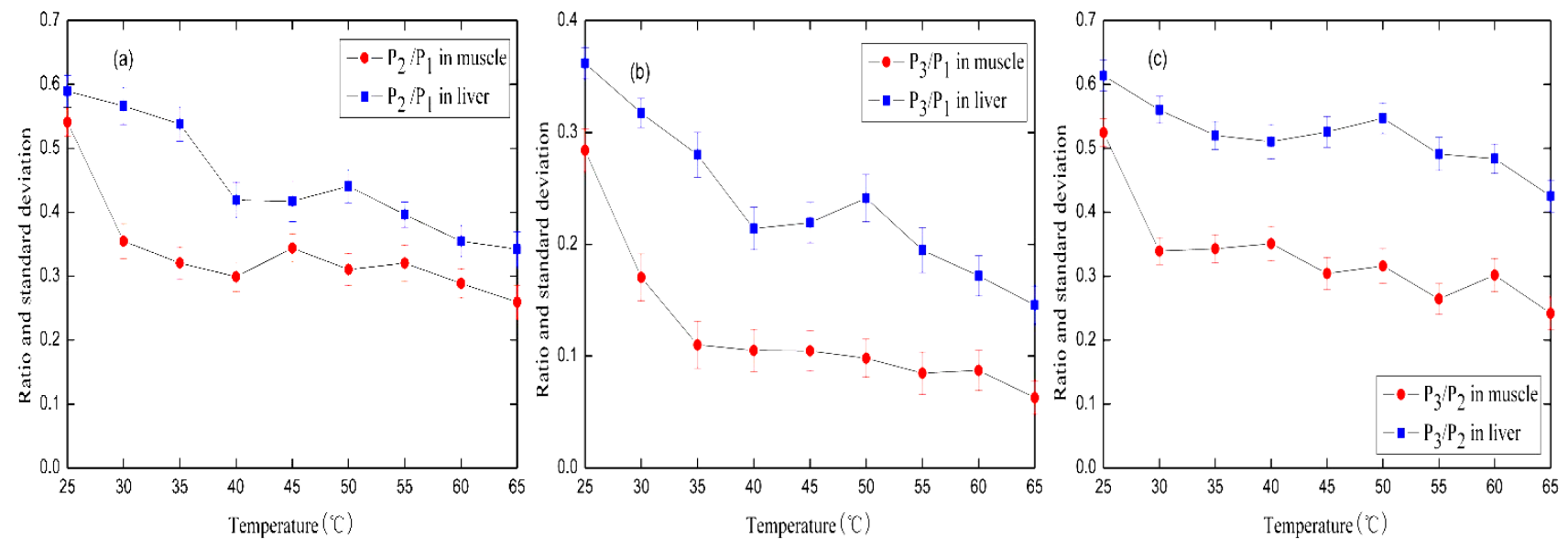

Fig. 5. Ratio and standard deviation of various harmonic amplitude at different temperature in porcine muscle and liver. (a) $P_{2} / P_{1}$, (b) $P_{3} / P_{1}$, (c) $P_{3} / P_{2}$.

\section{Discussion and conclusions}

In this paper, the relationship between scattering harmonics and temperature in the focal region of porcine muscle and liver was studied. Following conclusions can be obtained through our research:

1. The nonlinear harmonics generated in porcine muscle and liver are correlated with the change of temperature, and the temperature has certain effect on harmonic amplitude.

2. As temperature increases in the focal region of porcine muscle and liver, the difference of variation rate of harmonic amplitude decreases in porcine muscle and liver.

3. The slope of $P_{2} / P_{1}, P_{3} / P_{1}$, and $P_{3} / P_{2}$ have obvious decline at the beginning of temperature measurement, but their slope become flat as temperature increases. The slope of $P_{2} / P_{1}, P_{3} / P_{1}$, and $P_{3} / P_{2}$ drop faster in porcine muscle than in liver.

4. The amplitude variation of $P_{1}, P_{2}$, and $P_{3}$ associated with temperature is correlated with the affection of nonlinearity and absorption.

On the basis of this study, further testing on in vivo or perfused tissue is required to verify the relationship between harmonic amplitude and temperature. We used the change of harmonic amplitude to measure temperature, which does not need another source and could reduce damage to normal tissue caused by excessive thermal dose. This study shows us a potential approach to monitor the process of HIFU treatment.

\section{Acknowledgments}

This work was supported by the National Natural Science Foundation of China under grant No. 11474090, 11774088, and Scientific Research Fund of Hunan Provincial Education Department of China under grant No. $17 \mathrm{~B} 025$.

\section{References}

[1] J. Huang, R.G. Holt, R.O. Cleveland, R.A. Roy, J. Acoust. Soc. Am. 116, 2451 (2004).

[2] V. Rieke, P.K. Butts, J. Magn. Reson. Imag. 27, 376 (2008).

[3] N.M. Hijnen, E. Heijman, M.O. Köhler, M. Ylihautala, G.J. Ehnholm, A.W. Simonetti,H. Grüll, Int. J. Hyperther. 28, 141 (2012).

[4] P. Rangraz, H. Behnam, P. Sobhebidari, J. Tavakkoli, Ultrasound Med. Biol. 40, 2841 (2014).

[5] N.P. Brodin, A. Partanen, P. Asp, C.A. Branch, C. Guha, W.A. Tomé, Med. Phys. 43, 1167 (2016).

[6] D. Melodelima, V. Barrere, J. Acoust. Soc. Am. 137, 2399 (2016).

[7] C. Yang, H. Zhu, S. Wu, Y. Bai, H. Gao, J. Ultrasound Med. 29, 1787 (2010).

[8] S.Y. Shalhoub, A.A. Badr, J. Acoust. Soc. Am. 20, 1028 (2006)

[9] Z. Zhang, T. Chen, D. Zhang, Chin. Phys. Lett. 30, 024302 (2013).

[10] K.W. Van Dongen, M.D. Verweij, Int. J. Hyperther. 27, 612 (2011).

[11] W. Shou, X. Huang, S. Duan, R. Xia, Z. Shi, X. Geng, F. Li, Ultrasonics 44, e17 (2006).

[12] M. He, Z. Zhong, X. Li, X. Gong, Z. Wang, F. Li, Ultrason. Sonochem. 36, 36 (2017). 
[13] J.C. Bamber, Ultrasonic Properties of Tissues, IOP Pub., Bristol 1998.

[14] C.A. Damianou, N.T. Sanghvi, F.J. Fry, R. Maassmoreno, J. Acoust. Soc. Am. 102, 628 (1997).

[15] W. Keck, R.T. Beyer, Phys. Fluids 3, 346 (1960).

[16] F.A. Duck, Ultrasound Med. Biol. 28, 1 (2002).

[17] S. Zhang, M. Wan, H. Zhong, C. Xu, Z. Liao, H. Liu, S. Wang, Ultrasound Med. Biol. 35, 1828 (2009).

[18] J.C. Min, S.R. Guntur, J.M. Lee, G.P. Dong, I.L.L. Kang, A. Coleman, Ultrasound Med. Biol. 37, $2000(2011)$
[19] V. Zderic, A. Keshavarzi, M.A. Andrew, S. Vaezy, R.W. Martin, Ultrasound Med. Biol. 30, 61 (2004).

[20] T. Koch, S. Lakshmanan, S. Brand, M. Wicke, K. Raum, D. Mörlein, Meat Sci. 88, 67 (2011).

[21] I.M. Hallaj, R.O. Cleveland, K. Hynynen, J. Acoust. Soc. Am. 109, 2245 (2001).

[22] A. Payne, R. Merrill, E. Minalga, U. Vyas, B.J. De, N. Todd, R. Hadley, E. Dumont, L. Neumayer, D. Christensen, R. Roemer, D. Parker, Med. Phys. 39, 1552 (2012). 